\section{Does relaxation on a bed of nails (spike mat) induce beneficial effects? A randomized controlled pilot study}

\author{
Anette Kjellgren,1,2 Kristin Erdefelt,1,2 \\ Lena Werngren, ${ }^{3}$ Torsten Norlander ${ }^{1}$ \\ 1Human Performance Laboratory, \\ Karlstad University, Karlstad; \\ 2Department of Psychology, Karlstad \\ University, Karlstad; \\ 3Pain Clinic, Central Hospital of Karlstad, \\ County Council of Värmland, Karlstad, \\ Sweden
}

\section{Abstract}

Self-care treatments with bed of nails or spike mats have gained increased popularity world-wide; advertised as a method for pain reduction and wellbeing. Scientific studies regarding effects are still lacking. The aim of the present study was to investigate if daily relaxation on a spike mat for three weeks could induce beneficial effects. Participants were 36 individuals suffering from muscle tension pains in their back or/and neck. They were randomly assigned to a control group or an experimental group, who were treated with 15 minutes daily rest during three weeks on the spike mat. Significant reduced experienced worst pain intensity was found. There were no effects on normal pain intensity, optimism, anxiety, depression, stress, energy, or sleep quality. The participants appreciated the treatment, but their enthusiastic verbal reports of experienced beneficial effects could not be verified in the statistical analyses. The reduction of worst pain may be explained by the gate-control theory, where competing stimuli applied over the affected area produce a pain reduction. It can also be an effect of placebo or the relaxation. More research on relaxation on a spike mat is needed before its possible effects can be confirmed. No negative effects were found in the present study, but it has to be remembered no studies investigating risks for treatment on spike mats exist.

\section{Introduction}

In Sweden, self-care treatments with bed-ofnails or spike mats have became very popular. It has been estimated several hundred thousand spike mats have been sold last years in Sweden, ${ }^{1}$ and the interest still seems to be on the rise in several countries. Spike mats are sold by e.g. health food stores and massage therapists but recently sale has spread to also include e.g. supermarkets and petrol stations. Also several web shops are selling different brands of spike mats (we have found at least 20 different brands being sold in Sweden). Advertisements for spike mats promises alleviation from many different symptoms like pains, sleep disorders, depression, migraine and several others; statements without any scientific base. Treatments with spike mats are also advertised as an effective method for reducing stress. As far as we know, no scientific studies regarding possible effects or safety with the use of this device exist.

Stress-related illnesses such as fatigue, chronic pain, depression and sleep-disorders have increased in number and frequency in recent decades in western societies, ${ }^{2}$ so there is certainly a demand for stress reducing methods. A multitude of such methods exists like yoga, tai chi, chi gong, meditation, and flotation tank. ${ }^{3-10}$ Common to all these methods is that they besides beneficial health effects also can induce altered states of consciousness (ASC) with changes in e.g. cognitive functions, altered sense of time, and sometimes also mystical or ineffable experiences. ${ }^{11}$

Treatments in flotation tank (sensory isolation) is a method investigated by us during the last decade, and this treatment have been found to generate a multitude of positive effects like inducing deep relaxation and stress reduction, increased sleep quality, significantly reducing stress-related pain, increasing optimism, and decreasing the degree of depression and anxiety. ${ }^{12-16}$ During this work we first came into contact with spike mats. Several patients at our stress clinic spontaneously mentioned they have tried spike mats for relaxation and pain reduction. Some of them also brought their spike mats to the clinic for use before they entered into the flotation tank; they stated it helped them to relax even more during the subsequent flotation tank treatment.

Spike mats have sharp spikes (or nails), usually made in plastic. Lying down on the spike mat does not hurt the skin, but induce feelings of pain or at least feelings of sensory overload of sharp pressure. Manufacturers of spike mats usually recommend $15-40$ minutes of treatment time.

Despite extensive search in databases we have not found any other study investigating spike mats. In order to get more knowledge about this treatment the present study was designed. If this seems to be a promising area, our intentions are later performing a randomized controlled trial with a larger sample; but first we would like to make a small scale pilot study, in order getting more information. Two different spike mats were selected, with the
Correspondence: Anette Kjellgren, Department of Psychology, Karlstad University, SE-651 88 Karlstad, Sweden.

Tel. +46.547002173 - Fax: +46.547001460

E-mail: anette.kjellgren@kau.se

Key words: relaxation, spike mat, bed of nails, pain.

Contributions: LW, study patients management; $\mathrm{KE}$, data collection, spike mats distribution and visits booking; AK, manuscript design and participants recruitment; TN, manuscript design and statistical analysis.

Funding: the study was approved by the Ethical Board on Experimentation on Human Subjects (Regionala Etikprövningsnämnden) in Uppsala, Sweden (Dnr 2009/149). The study was supported by grants from the County Council in Värmland (Landstinget - LiV), Sweden.

Conflict of interest: the authors report no conflicts of interest. No financial support from any manufacturer of spike mats has been received. The choice of the two brands of spike mats were our own decision and not due to any demand from any company.

Received for publication: 10 February 2011. Accepted for publication: 25 March 2011.

This work is licensed under a Creative Commons Attribution 3.0 License (by-nc 3.0).

CC Copyright A. Kjellgren et al., 2011

Licensee PAGEPress, Italy

Alternative Medicine Studies 2011; 1:e5

doi:10.4081/ams.2011.e5

demands to be easily cleaned and hygienic, and produced in an allergy friendly plastic material. These two mats were selected by us, based on the aforementioned demands, and were not due to any desires from any company or manufacturer. No financial support from manufacturers has been received.

The aim of the present study was to get information whether or not relaxation on a spike mat could induce beneficial effects for persons suffering from muscle tension pains in their back and/or neck.

\section{Materials and Methods}

\section{Participants}

Thirty-six participants (10 men and 26 women) were included in the study. They all reported sufferings from muscle tension pains in their neck or/and back. They reported having had pain for an average of 15.88 years (SD=16.26, range:0.33-60 years) and 22 of the persons took analgesics on a regular basis. The 
average age of the participants were 54.44 years $(\mathrm{SD}=15.15$, range $=21-81$ years $)$. All participants were spike mat naïve and had not tried this method before. Twenty persons interested in participating in the study were excluded due to earlier experience with spike mat. Inclusion criteria were: experienced suffering from stress related pain in the back and/or neck, and to be spike mat naïve. Exclusion criteria were pregnancy or breast-feeding, pacemaker, open wounds or skin irritations, psychiatric disorder, or use of anticoagulants (one person excluded due to this). If significant discomfort occurred during treatment this was also considered as exclusion criteria (no person was excluded due to this). Participants were initially recruited from the Pain Clinic, Central Hospital of Karlstad, County Council Värmland, Karlstad, but since most pain patients there already used a spike mat as self care treatment at home, the recruitment process were extended to include advertisements at health care centres in Värmland and also from a local radio program.

The participants were randomly assigned to a control group or an experimental group. The experimental group (spike mat) constituted of 21 persons, and the control group (no intervention) of 15 persons. A total of 18 persons were initially randomized to the control group, but three immediately withdraw their participation without generating any data. These three persons were not included in the analysis. The 21 persons in the experimental group were then further randomized to use one of two different brands of spike mats (green: 10 persons, or white: 11 persons). See Figure 1 for a flow diagram of the process.

There were no differences between the control group and the experimental group (independent samples t-test, ps $>0.14$ ) regarding age, duration of pain, use of analgesics, having pain in the back or neck, and consumption of cigarettes or alcohol.

\section{Design}

This study used a two-way split-plot design, where Time with assessment before and after the treatment period constituted the withinsubjects factor and where Group (control, spike mat) constituted the between subject factor. The treatment time was three weeks, and the participants in the experimental group visited the laboratory on three occasion (before, during second week, after three weeks), and the control group made two visits (before, after). The protocol for this study was approved by the Ethical Board on Experimentation on Human Subjects (Regionala Etikprövningsnämnden) in Uppsala, Sweden (Dnr 2009/149). All participants gave written informed consent for participation.

\section{Procedure}

When accepting participation in the project, each client signed a written agreement regarding their guarantee of confidentiality and their right to terminate whenever they wanted. Further, the written agreement determined the collected material could only be used as a basis for publication on the condition that the confidentiality of the client was guaranteed.

Upon arrival at the first session quantitative data were gathered using the questionnaires HAD, LOT, SE, PAI, Pain-VAS, as well as background data about age, gender, duration of pain, ongoing medication, and consumption of alcohol and cigarettes (see Instruments). For persons in the control group a new time for a final visit three weeks later was booked. If the person belonged to the experimental group, they thereafter performed the first treatment on the spike mat for 15 minutes, and were instructed to immediately terminate if they felt severe discomfort. The spike mat was on a couch and the participant gently laid down on his/her back (shirtless); the spike mat covered an area from the neck/shoulder down to the lumbar region. A blanket was provided for covering the front side of the body for increased warmth and comfort. After this first treatment the short scale Discomfort and pain induced by the spike mat were filled out (a value above 70 served as an exclusion criterion, no person made such estimation). A simple calendar to enter the days the spike mat were used were distributed, together with the instruction to use the mat 15 minutes at least five out of seven days in a week. Two other visits were booked (one the second week and a final visit three weeks later), and a phone number was distributed in case of any questions later. The spike mat was then taken home for use. At arrival for the second visit (experimental group only) participants were asked if there were any problems or questions. Directly after a 15 minutes treatment on the spike mat the Discomfort and pain induced by the spike mat scale, as well as the EDN-scale was filled out. The use of the daily calendar was also checked for. At the third and final visit the same procedure as for the second visit followed, with the addition of distribution of the instruments HAD, LOT, SE, PAI, and Pain-VAS (same as during the first visit). The spike mat was collected and participants were thanked for their participation. During the last (and second) visit for the control group the instruments HAD, LOT, SE, PAI, and Pain-VAS were filled out and they were also thanked for their participation as well.

\section{Instruments}

\section{Spike mats}

Two different brands of spike mats were used. The first one (green) was of the brand Svenska spikmattan (The Swedish spike mat, http://www.spikmattan.com) with measure-

\section{Enrollment}

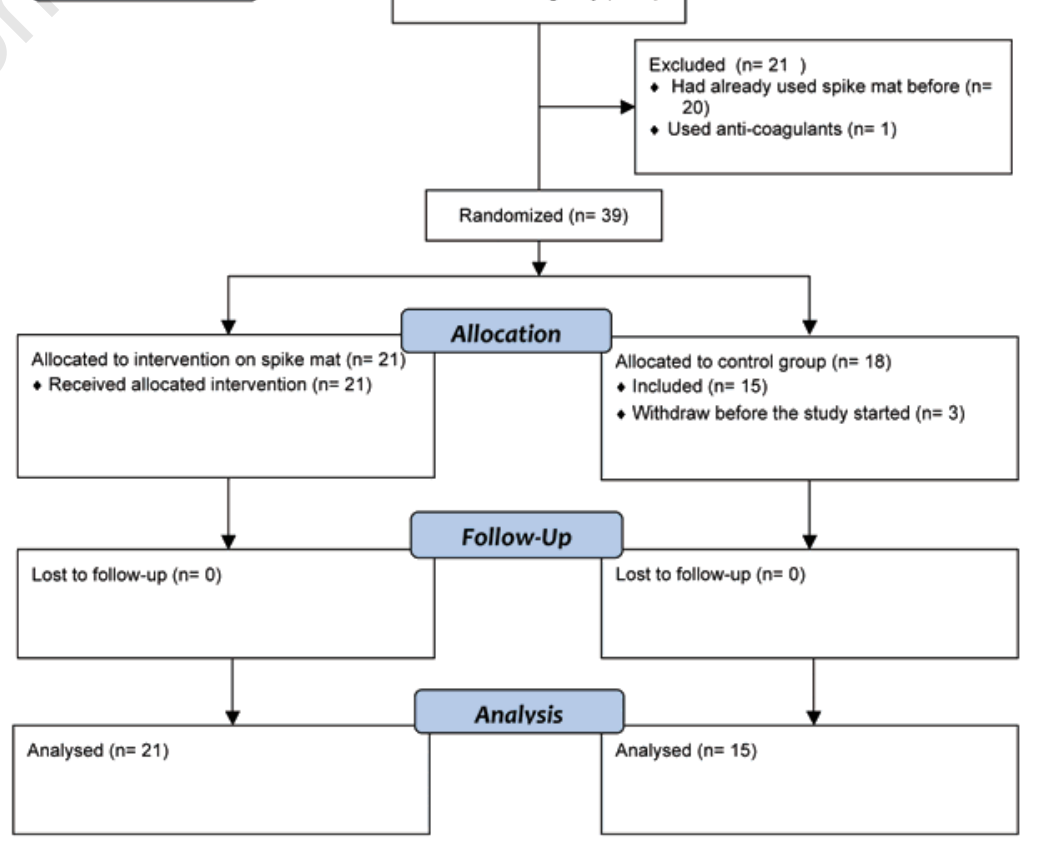

Figure 1. Flow diagram depicting the process. Template used for the flow diagram was downloaded from http://www.consort-statement.org/consort-statement/flow-diagram0/.17 
ments $40 \times 60 \mathrm{~cm}$ and equipped with 600 nails, each of $15 \mathrm{~mm}$ length. The second one (white) was of the brand Rysk spikmatta (The Russian spike mat, http://www.ryskspikmatta.se) with measurements $68 \times 38 \mathrm{~cm}$ and with a total of 4158 nails, each with a length of $4 \mathrm{~mm}$. The participants used the spike mat each day (15 minutes) during the three weeks period; the use of the spike mat was checked for with a short calendar.

\section{Hospital anxiety depression scale}

The HAD is a rating scale concerning degree of anxiety and depression, and was constructed by Zigmond and Snaith. ${ }^{18}$ It has since been revised to be used as a rating scale for anxiety and depression. ${ }^{19}$ The instrument consists of fourteen statements with four response alternatives (i. e., 0 to 3), ranging from positive to negative or vice versa. There are seven statements regarding anxiety and seven regarding depression. The HAD-scale was completed before and after the treatment period.

\section{Life orientation test}

The test ${ }^{20}$ consists of eight items, plus four filler items. The task of each participant is to decide whether or not one is in agreement with each of the items described, on a scale of 0-4, where 0 indicates strongly disagree and 4 indicates strongly agree. The test measures dispositional optimism, defined in terms of generalized outcome expectancies. Parallel Test Reliability is reported to 0.76 and Internal Consistency to 0.76 and Test-Retest reliability to 0.75 .21

\section{Stress and energy}

The stress and energy (SE) instrument is an instrument concerning individuals' energy and stress experiences. ${ }^{22}$ The scale consists of two subscales that elucidate the mood levels of the subjects on the dimensions: experienced stress and experienced energy. The response alternatives were arranged on six-grade scales, extending from: $0=$ not at all, to $5=$ very much. The instrument has been validated by analyses from studies focused on occupational burdens and pressures. ${ }^{23}$ There was no time limit for this test.

\section{Pain area inventory}

The test consists of two anatomical images of a human being, one frontal and one dorsal.13,24 The task of the participants was to indicate with a color pen their areas of pain. This test was given both before and after the treatment period. For scoring, a transparent, plastic film is then placed over the colored areas on both figures. Each figure is divided into 833 equal-sized squares (total 1666), and the number of colored squares was calculated. The test was validated 13 through comparisons with other instruments by examining relationships with total number of pain types reported, number of connected pain areas, most severe pain intensity, normal pain intensity, and pain frequency. The data yielded acceptable values (Standardized item alpha $=0.84, \mathrm{R}=0.70$ ). TestRetest reliability was examined through using a group of pain patients who completed the PAI on two occasions, seven weeks apart $(\mathrm{r}=0.92)$.

\section{Normal pain and worst pain - visual ana- logue scale}

These scales were used for measuring normal pain and experienced worst pain. They consisted of a $100 \mathrm{~mm}$ horizontal line with the anchors no pain on the left extreme and excruciating pain on the right extreme. VAS (visual analogue scale) is considered the gold standard for assessment of clinical pains, and changes in VAS score are regarded as significant evidence of response to treatment, placebo, or experimental manipulation. ${ }^{25}$ The accuracy and precision have been examined for both clinical and experimental pain, and found adequate. ${ }^{26}$

\section{Sleep quality}

This instrument consists of 11 questions that tap the sleeping habits of the participants, such as How often do you feel tired on week days?, How often do you feel you did not get enough sleep?, How do you feel you usually sleep? Responses to 9 of the questions are given on $0-4$ scales, to 1 question on a $0-5$ scale, and finally to 1 question on a 0-8 scale. The psychometric properties were examined by comparing healthy and sick people, and using Cronbach's alpha $(\alpha=0.88) .27$

Discomfort and pain induced by the spike mat

This was a VAS aimed at assessing experienced pain or discomfort induced by the spike mat during treatment. It consisted of a $100 \mathrm{~mm}$ horizontal line with the anchors $0=$ no pain or discomfort on the left extreme and $100=$ excruciating pain or discomfort on the right extreme. A value of 70 or above was considered an exclusion criterion (no participant was excluded due to this).

\section{Experienced deviation from normal state}

Utilizing the internationally applied psychometric instruments APZ-questionnaire and OAVAV28 for obtaining judgments of altered states of consciousness, a shortened but similar instrument was modified for use with flotation tank therapy. 14 The original tests have been validated in several studies over different countries. ${ }^{28}$ The shortened version utilized in the present study, consists of 29 questions, each followed by a VAS $(100 \mathrm{~mm})$. All points from the 29 items were averaged to form an index of experience reflecting the total experience of deviation from normal states. The instrument acquired judgments of altered states of consciousness (ASC). There is a strong connection between altered state of consciousness and the relaxation response. ${ }^{29}$

\section{Results}

\section{Pain measurements}

The analyses were performed with two-way split plot ANOVAS, where Time with assessment before and after the treatment period as the within-subjects factor and with Group (control, spike mat) as the between subject factor. The analysis Discomfort and pain induced by the spike mat (for the experimental group only) was performed with a repeated measure ANOVA with Time (first treatment, during second week, last treatment) as the within-subject variable.

\section{Worst pain intensity \\ The analysis yielded a significant difference} for Time $\left[\mathrm{F}_{(1.34)}=8.16, \mathrm{P}=0.007\right.$; eta $^{2}=0.193$, power $=0.792]$ where the degree of worst pain intensity diminished from $67.64(\mathrm{SD}=17.16)$ to $58.39(\mathrm{SD}=22.83)$ during the treatment time. There was a significant interaction between Time and Group $\left[\mathrm{F}_{(1.34)}=4.48\right.$, $\mathrm{P}=0.042$; eta ${ }^{2}=0.116$, power $\left.=0.538\right]$ where the experimental group significantly reduced their worst pain intensity from 68.14 (SD=17.26) pre-treatment to 53.81 ( $\mathrm{SD}=24.37)$ post treatment, while control group had a worst pain intensity of $66.93(\mathrm{SD}=17.60)$ at pre-treatment and had no significant alteration at post treatment test $(M=64.80, S D=19.45)$. See Table 1 for means and standard deviations.

\section{Normal pain intensity}

There were neither significant effects nor interactions regarding normal pain intensity (ps>0.58) (Table 1).

\section{Pain-area inventory \\ There was a significant decrease in number of painful areas for Time $\left[\mathrm{F}_{(1.34)}=9.47\right.$,}

Table 1. Pain measurements with means and (standard deviations) before and after the three week treatment period.

\begin{tabular}{lcccc} 
Variable & \multicolumn{2}{c}{$\begin{array}{c}\text { Control group } \\
\text { Before }\end{array}$} & $\begin{array}{c}\text { After } \\
\text { Before }\end{array}$ & $\begin{array}{c}\text { Experimental group } \\
\text { After }\end{array}$ \\
Worst pain intensity & $66.93(17.60)$ & $64.80(19.45)$ & $68.14(17.26)$ & $53.81(24.37)$ \\
Normal pain intensity & $28.00(19.77)$ & $28.20(18.42)$ & $30.24(16.55)$ & $28.05(17.55)$ \\
\hline Painful areas (PAI) & $74.53(84.30)$ & $61.20(58.91)$ & $70.57(52.93)$ & $50.10(43.45)$ \\
\hline
\end{tabular}


$\mathrm{P}=0.004 ;$ eta $^{2}=0.218$, power $=0.848$ ] from 72.2 $(\mathrm{SD}=66.69)$ pre-treatment to $54.72(\mathrm{SD}=$ $54.72)$ post treatment. There was no significant differences between the groups $(\mathrm{P}=0.70)$ (Table 1).

Discomfort and pain induced by the spike mat

These analyses were only performed for the experimental group. There were no significant differences for Time $(\mathrm{P}=0.057)$. However, there was a significant linear trend $\left[\mathrm{F}_{(1.20)}\right.$ $=5.48, \mathrm{P}=0.030 ;$ eta $^{2}=0.215$, power $=0.606$ ], where the experienced discomfort diminished from $14.62(S D=16.10)$ for the first treatment, to $10.95(\mathrm{SD}=10.44)$ at treatment during second week, and to $9.76(\mathrm{SD}=9.60)$ for the last treatment.

\section{Psychological variables}

The analyses were performed with two-way split plot Pillai's MANOVA, where Time with assessment before and after the treatment period constituted the within-subjects factor and where Group (control, spike mat) constituted the between subject factor. The dependent variables were depression, anxiety, optimism, stress, energy, and sleep quality.

There were no significant effects of Time $\left(\mathrm{P}=0.158\right.$; eta $\left.^{2}=0.260\right)$ or Group $(\mathrm{P}=0.124$; eta $\left.^{2}=0.277\right)$ or interactions $\left(\mathrm{P}=0.679\right.$; eta $^{2}=$ 0.121 ). The $\mathrm{p}$-values from the univariate $\mathrm{F}$ tests concerning Time, Group and interactions Time $\mathrm{x}$ Group were: depression ( $\mathrm{ps}>0.175$ ), anxiety (ps $>0.171)$, optimism (ps $>0.392)$, stress (ps>0.075), energy ( $p s>0.370)$ and sleep quality (SQ) (ps>0.144). See Table 2 for means and standard deviations. Degrees of experienced degree of altered states of consciousness (as measured with the EDN-scale for the experimental group) were $\mathrm{M}=18.79$ $(\mathrm{SD}=14.91)$ direct after treatments during the second week, and $\mathrm{M}=15.67$ ( $\mathrm{SD}=14.12$ ) after the last treatment the third week. There were no significant differences between these values (paired-samples t-test, $\mathrm{P}=0.27$ ).

\section{Discussion}

The aim of the present study was to get information whether or not relaxation on a spike mat could induce beneficial effects for persons suffering from muscle tension pains in their back and/or neck. To our best knowledge, no other studies have been performed dedicated to study possible effects of this device. The main findings of the present study was that 15 minutes daily rest (during three weeks) on the spike mat significantly reduced the experienced worst pain intensity, but did not affect the normal pain intensity. No other significant effects were found in the present study. Nor were there any differences in

Table 2. Psychological variables with means and (standard deviations) before and after the three week treatment period.

\begin{tabular}{|c|c|c|c|c|}
\hline \multirow[t]{2}{*}{ ariable } & \multicolumn{2}{|c|}{ Control group } & \multicolumn{2}{|c|}{ Experimental group } \\
\hline & Before & After & Before & After \\
\hline Depression & $2.20(2.21)$ & $2.73(2.37)$ & $2.76(2.49)$ & $2.43(2.42)$ \\
\hline Anxiety & $4.40(2.53)$ & $5.13(3.56)$ & $6.33(3.64)$ & $5.81(3.23)$ \\
\hline Optimism & $21.33(4.60)$ & $21.33(4.71)$ & $22.29(3.76)$ & $23.14(4.52)$ \\
\hline Stress & $2.44(0.73)$ & $2.22(0.85)$ & $2.08(0.86)$ & $1.91(0.96)$ \\
\hline Energy & $3.74(0.78)$ & $3.74(0.74)$ & $3.47(0.82)$ & $3.69(0.76)$ \\
\hline Sleep quality & $26.07(9.60)$ & $26.40(9.79)$ & $23.38(8.05)$ & $24.86(7.36)$ \\
\hline
\end{tabular}

effects between the two different spike mats used in the present study (these analyses were not presented in the results section, but it was checked for, ps $>0.117$ ). The participants in the present study reported having suffered from muscle tension pains for in average about 16 years. A significant reduction of worst pain intensity for persons suffering from apparently treatment resistant pains may be considered as promising, but of course no conclusions can be made from just one small scale pilot study.

The daily treatments on the spike mat did not significantly affect the measured psychological variables. There were some indications that depression, anxiety and stress diminished, as well as small increases in optimism and energy during the 3 week treatment period, but these were not statistically significant. Despite these non significant results, participants were very enthusiastic about the treatment. During their visits at the clinic they spontaneously stated they felt more energized, slept better at nights and that the spike mat had made them feel better, but these statements could not be verified in the statistical analyses. The sampling method used ought to be commented upon. The present study is small $(n=36)$ since we had problems recruiting spike mat naïve participants. There were about 20 other persons volunteering for participating, but they already used spike mats at home on a regular basis since, as they stated, it helps a lot as pain reduction. This means that persons who already have experienced possible beneficial effects were not included due to their earlier experience of spike mats.

Looking at the advertisements' for spike mats in media, there are lots of speculative references to yogis and spike mats as an ancient technique facilitating altered states of consciousness to emerge. We did not find that the spike mats (that were used in the present study) produced any notable degree of altered states of consciousness. The mean EDN-values (degree of altered states of consciousness) of about 15-18 during the treatment was similar to e.g. resting on a bed in a dark room ${ }^{30}$ or looking at peaceful slideshow, 31 implying just a modest difference from normal state of consciousness. The variable altered state of consciousness seems to be of importance for treat- ments of stress related ailments like muscle tension pains, anxiety and depression. Typical EDN-value for relaxation during flotation tank treatments are $30-40$ points. ${ }^{30}$ Another method involving altered states of consciousness is Sudarshan Kriya Yoga (typical value is about 40) where beneficial effects like diminished anxiety, depression, stress, as well as increased optimism and energy seems to occur. ${ }^{3}$ It may be speculated the altered states of consciousness induced during relaxation exercises enables activation of self-healing processes in the mind and body.

Nevertheless, the participants were enthusiastic and liked the treatments on the spike mats. Maybe the sensory overload (discomfort or pain) on the spike mat served as a trigger for inducing a state of here-and-now or mindfulness. During such a state, abstract thoughts and ideas about problems or things that have happened or might happen (then-and-then thinking) decreases, thereby implying a sense of wellbeing. It may be restful and psychologically beneficial to experience recurring episodes of the just be state. If the spike mat could be used as a complementary tool for facilitation of mindfulness meditation or stress reduction could be an interesting future application. It could also be argued that the process of just lying down relaxing may induce a sense of well-being.

The temporary discomfort or pain experienced during the relaxation on the spike mat (although quite mild) seemed to decrease over time. The reduction of worst pain documented in the present study may be explained by the gate-control theory, 32 where competing stimuli applied over the affected area produce a temporary pain relief. The effects of increased blood flow due to the sharp spikes may also be a possible explanation for pain reduction. For persons suffering from chronic pain, the possibility of gaining some degree of control of their pains may have a stress-reducing effect with a positive outcome for experienced pains. Effects due to the spike mats' influence on acupuncture points or meridians can not be assessed in this study. Neither is it from this study possible to determine if any changes in levels of e.g. endorphins, endocannabinoids, or oxytocin contributed to the pain reducing 
effect. The importance of placebo effects should not be underestimated either.

Obviously the participants experienced the spike mat as a suitably method for cutting temporary pain peaks, even though most of promises provided by advertisements could not be verified. Our conclusion is that more research is needed before the possible effects of spike mats can be confirmed.

Suggestions for studies in the future could be using a larger sample and with other ailments, longer treatment periods, as well as follow-up periods. A further study should preferably also include some sort of intervention for the control condition (e.g. relaxation on a yoga mat or on a common mattress). Another possible design consideration would be to provide both groups with some sort of intervention, such as exercise, and then one group with a spike mat. Also a careful mapping of neurobiological and hormonal correlates in blood samples before and after treatments would be of great interest to further investigate possible effects or mechanisms of spike mats, if such exist. No negative effects were noted in the present study. It has to be remembered no studies investigating safety for persons suffering for cardio-vascular diseases or serious illnesses has been performed. Although, since the spike mat seems to gain more and more popularity as a self care treatment method, any risks or exclusion criteria for treatment also deserves close attention.

\section{References}

1. Dagens medicin [Medicine of today], [Internet, retrieved 16 Aug 2010]. Available $\begin{array}{llll}f & r & 0 & m\end{array}$ http//www.dagensmedicin.se/nyheter/2009/ $07 / 24 /$ k 0 - a g e r a r - mot - f a ls kreklam/index.xml

2. Folkow B. Evolution och fysiologi [Evolution and physiology]. In: Ekman R, Arnetz B, editors. Stress: Individen, samhället, organisationen och molekylerna [Stress: The individual, society, the organisation and the molecules]. Stockholm: Liber, 2005. p. 29-45.

3. Kjellgren A, Bood SÅ, Axelsson K, et al. Wellness through a comprehensive Yogic Breathing Program - A controlled pilot trial. BMC Complement Altern Med 2007; 7:43.

4. Shapiro D, Cook IA, Davydov DM, et al. Yoga as a complementary treatment of depression: Effects of traits and moods on treatment outcome. Evid Based Complement Alternat Med 2007;4:493-502.

5. Sandlund ES, Norlander T. The effects of Thai Chi Chuan relaxation and exercise on stress responses and well-being: an overview of research. Int J Stress Manag
2000;7:139-49.

6. Craske NJM, Turner W, Zammit-Maempe J, Lee MS. Qigong ameliorates symtoms of chronic fatigue: A pilot uncontrolled study. Evid Based Complement Alternat Med 2009;6:265-70.

7. Kjellgren A, Taylor S. Mapping Zazen Meditation as a Developmental process: Exploring the experiences of experienced and inexperienced meditators. J transpersonal psy 2008;2:224-50.

8. Hankey A. Studies of advanced stages of meditation in the Tibetan Buddhist and vedic tradtions. I: A comparison of general changes. Evid Based Complement Alternat Med 2006; 3:513-21.

9. Kjellgren A. The experience of flotationREST (Restricted Environmental Stimulation Technique): Consciousness, creativity, subjective stress, and pain [dissertation]. Sweden: Gothenburg University; 2003.

10. Bood SÅ. Bending and mending the neurosignature. Frameworks of influence by flotation-REST (Restricted environmental stimulation technique) upon well-being inpatients with stress related ailments [dissertation]. Sweden: Karlstad University;2007.

11. Kjellgren A, Lydén F, Norlander T. Sensory isolation in flotation tank: altered states of consciousness and effects on well-being. The Qualitative Report 2008;13:636-56.

12. van Dierendonck $\mathrm{D}$, te Nijenhuis $\mathrm{J}$. Flotation restricted environmental stimulation therapy (REST) as a stress management tool for enhancing well-being and performance: A meta-analysis. Psychol Health 2005;20:405-12.

13. Bood S A, Sundequist U, Kjellgren A, et al. Effects of flotation-REST (Restricted Environmental Stimulation Technique) on stress related muscle pain: What makes the difference in therapy, attention-placebo, or the relaxation response? Pain Res Manag 2005;10:201-9.

14. Kjellgren A, Sundequist U, Norlander T, Archer T. Effects of flotation-REST of muscle tension pain. Pain Res Manag 2001;6: 181-9.

15. Bood S A, Sundequist U, Norlander T, et al. Eliciting the relaxation response with help of flotation-REST (Restricted environmental stimulation technique) in patients with stress related ailments: The direct effects and effects four months after treatment. Int J Stress Manage 2006;13:154-75.

16. Åsenlöf K, Olsson S, Bood SÅ, Norlander T. Case studies on fibromyalgia and burn-out depression using psychotherapy in combination with flotation-REST: Personality development and increased well-being. Imagination, Cognition, and Personality 2007;26:259-71.
17. Moher D, Schulz KF, Altman DG. The CONSORT statement: revised recommendations for improving the quality of reports of parallel-group randomized trials. Ann Intern Med 2001;134:657-62.

18. Zigmond S, Snaith RP. The hospital anxiety and depression scale. Acta Psychiatr Scand 1983;67:361-70.

19. Herrmann C. International experiences with the hospital anxiety and depression scale. A review of validation data and clinical results. J Psychosom Res 1997;42:17-41.

20. Scheier MF, Carver CS. Optimism, Coping and Health: assessment and Implications of Generalized Outcome Expectancies. Health Psychol 1985;4:219-47.

21. Norlander T, Bergman H, Archer T. Relative constancy of personality characteristic and efficacy of a 12-month training program in facilitating coping strategies. Soc Behav Pers 2002;30:773-84.

22. Kjellberg A, Iwanowski S. Stress/energi formuläret: Utveckling av en metod för skattning av sinnesstämning i arbetet [The Stress/arousal questionnaire: Development of a method for assessment of mood at work]. Solna, Sweden: Arbetsmiljöinstitutet;1989.

23. Kjellberg A, Bohlin A. Self-reported arousal: Further development of a multi-factorial inventory. Scand J Psychol 1974;15:285-92.

24. Landström A, Bood SÅ, Kjellgren A, Norlander T. Treating stress related pain in a clinical sample with flotation-REST: A further report on improvements on pain assessed by the Pain Area Inventory (PAI). Soc Behav Pers 2007;35:1279-80.

25. Yarnitsky D, Sprecher E, Zaslansky R, Heimli A. Multiple session experimental pain measurement. Pain1996;67: 327-33.

26. Price, DD. Psychological and neural mechanisms of Pain. New York: Raven Press; 1988.

27. Norlander T, Johansson $\AA$, Bood SA. The affective personality: Its relation to quality of sleep, well-being and stress. Soc Behav Pers 2005;33:709-22.

28. Dittrich A. The standardized psychometric assessment of Altered States of Consciousness (ASCs) in Humans. Pharmacopsychiatry 1998;31:80-84.

29. Benson $H$. The relaxation response. New York: Harper Collins; 2000.

30. Kjellgren A, Sundequist U, Sundholm U, et al. Altered Consciousness in flotation-REST and chamber-REST: Experience of Experimental Pain and Subjective Stress. Soc Behav Pers 2004;32:103-16.

31. Kjellgren A, Buhrkall H. A comparison of the restorative effect of a natural environment with a simulated environment. J Environ Psychol 2010;30:464-72.

32. Melzack R. Pain - an overview. Acta Anesth Scand 1999;43:880-4. 